\title{
Automatic Abstraction without Counterexamples
}

\author{
Kenneth L. McMillan and Nina Amla \\ Cadence Design Systems
}

\begin{abstract}
A method of automatic abstraction is presented that uses proofs of unsatisfiability derived from SAT-based bounded model checking as a guide to choosing an abstraction for unbounded model checking. Unlike earlier methods, this approach is not based on analysis of abstract counterexamples. The performance of this approach on benchmarks derived from microprocessor verification indicates that SAT solvers are quite effective in eliminating logic that is not relevant to a given property. Moreover, benchmark results suggest that when bounded model checking successfully terminates, and the problem is unsatisfiable, the number of state variables in the proof of unsatisfiability tends to be small. In almost all cases tested, when bounded model checking succeeded, unbounded model checking of the resulting abstraction also succeeded.
\end{abstract}

\section{Introduction}

Abstraction is commonly viewed as the key to applying model checking to large scale systems. Abstraction means, in effect, removing information about a system which is not relevant to a property we wish to verify. In the simplest case, we can view the system as a large collection of constraints, and abstraction as removing constraints that are deemed irrelevant. The goal in this case is not so much to eliminate constraints per se, as to eliminate state variables that occur only in the irrelevant constraints, and thereby to reduce the size of the state space. A reduction of the state space in turn increases the efficiency of model checking, which is based on exhaustive state space exploration.

The first attempt to automate this simple kind of abstraction is due to Kurshan [12, and is known as iterative abstraction refinement. This method begins with an empty set of constraints (or a seed set provided by the user), and applies model checking to attempt to verify the property. If a counterexample is found, it is analyzed to find a set of constraints whose addition to the system will rule out the counterexample. The process is then repeated until the property is found to be true, or until a concrete counterexample is produced. To produce a concrete counterexample, we must find a valuation for the unconstrained variables, such that all the original constraints are satisfied.

A number of variations on this basic technique have appeared [15/22]. Some of the recent methods pose the construction of a concrete counterexample as a Boolean satisfiability (SAT) problem (or equivalently, an ATPG problem) and 
apply modern SAT methods [14] to this problem. A recent approach [6] also applies ILP and machine learning techniques to the problem of choosing which constraints to add to rule out an abstract counterexample in the case when a concrete counterexample is not found.

Another recent and related development is that of bounded model checking [3]. In this method, the question of the existence of a counterexample of no more than $k$ steps, for fixed $k$, is posed as a SAT problem. In various studies [74], SAT solvers have been found to be quite efficient at producing counterexamples for systems that are too large to allow standard model checking. The disadvantage of this approach is that, if a counterexample is not found, there is no guarantee that there do not exist counterexamples of greater than $k$ steps. Thus, the method can falsify, but cannot verify properties (unless an upper bound is known on the depth of the state space, which is not generally the case).

In this paper, a method is presented for automated abstraction which exploits an under-appreciated fact about SAT solvers: in the unsatisfiable case, they can produce a proof of unsatisfiability. In bounded model checking, this corresponds to a proof that there is no counterexample to the property of $k$ steps or fewer. Even though this implies nothing about the truth of the property in general, we can use this proof to tell us which constraints are relevant to the property (at least in the first $k$ steps) and thus provide a guess at an abstraction that may be used to fully verify the property using standard model checking methods. The method differs from the earlier, counterexample-based iterative abstraction approaches, in that counterexamples produced by the standard model checker are ignored. The abstraction is based not on refuting these counterexamples, but rather on proofs provided by the SAT solver. Thus, we will refer to it as proof-based abstraction. This approach has the advantage that it rules out all counterexamples up to a given length, rather than the single counterexample that the model checker happened to produce.

\section{$1.1 \quad$ Related Work}

The notion of proof-based abstraction has already appeared in the context of infinite state verification. Here, a finite state abstraction of an infinite state system is generated using as the abstract states the valuations of a finite set of first order predicates over the concrete state. The key to this method, known as predicate abstraction [18, is to choose the right predicates. An iterative abstraction method proposed by Henzinger et al. [20] uses a theorem prover to refute counterexamples generated by predicate abstraction. In the case when the counterexample is proved false, the proof is "mined" for new state predicates to use in predicate abstraction.

The technique presented here is similar to this method in spirit, but differs in some significant aspects. First, it applies only to finite state systems, and uses a SAT solver rather than a first order prover. Second, instead of choosing predicates to define the abstract state space, it merely chooses among the existing constraints to form the abstraction - the encoding of the state remains the same. 
Another related technique [16] uses a SAT solver to derive an abstraction sufficient to refute a given abstract counterexample. The abstraction is generated by tracing the execution of the SAT solver in a way that is similar to the method presented here. However, that method, like the earlier methods, still refutes one counterexample at a time, accumulating an abstraction.

The key difference between the methods of 2016] and the present one is that the present method does not use abstract counterexamples as a basis for refining the abstraction. Rather, it generates an abstraction sufficient to refute all counterexamples within a given length bound. Intuitively, the motivation for refuting all counterexamples at once is that a given abstract counterexample may be invalid for many reasons that are not relevant to the truth of the property being proved. In the present method, the abstraction is directed toward the property itself and not a single execution trace that violates it.

Another important difference is that the generated abstraction is not cumulative. That is, a constraint that is present in abstraction in one iteration of the algorithm may be absent in a later iteration. Thus, strictly speaking, the method cannot be viewed as "iterative abstraction refinement". In the counterexamplebased methods, an irrelevant constraint, once added to the abstraction, cannot be removed.

\subsection{Outline}

We begin in the next section by considering how a Boolean satisfiability solver can be extended to produce proofs of unsatisfiability. Then, in section 3, we introduce the proof-based abstraction method. Finally, in section 4, we test the method in practice, applying it to the verification of some properties of commercial microprocessor designs.

Benchmark results provide evidence for two significant conclusions: first, that SAT solvers are quite effective at isolating the parts of a large design that are relevant to a given property, and second, that if a property is true and bounded model checking succeeds, then in most cases unbounded model checking can be applied to an abstraction to prove the property in general.

\section{Extracting Proofs from SAT Solvers}

A DPLL-style SAT solver, such as CHAFF [14], is easily instrumented to produce proofs of unsatisfiability using resolution. This is based on the observation that "conflict clause" generation can be viewed as a sequence of resolution steps, following the so-called "implication graph". Readers familiar with SAT methods may find this observation quite trivial, and therefore may wish to skip this section. Otherwise, we will now define what is meant by a "proof of unsatisfiability", and show how one can be extracted from a run of a typical SAT solver.

To begin at the beginning, a clause is a disjunction of a set of zero or more literals, each of which is either a Boolean variable or its negation. We assume that clauses are non-tautological, that is, no clause contains both a variable and 
its negation. A set of clauses is said to be satisfiable when there exists a truth assignment to all the Boolean variables that makes every clause in the set true.

Given two clauses of the form $c_{1}=v \vee A$ and $c_{2}=\neg v \vee B$, we say that the resolvent of $c_{1}$ and $c_{2}$ is the clause $A \vee B$, provided $A \vee B$ is non-tautological. For example, the resolvent of $a \vee b$ and $\neg a \vee \neg c$ is $b \vee \neg c$, while $a \vee b$ and $\neg a \vee \neg b$ have no resolvent, since $b \vee \neg b$ is tautological. It is easy to see that any two clauses have at most one resolvent. The resolvent of $c_{1}$ and $c_{2}$ (if it exists) is a clause that is implied by $c_{1} \wedge c_{2}$ (in fact, it is exactly $(\exists v)\left(c_{1} \wedge c_{2}\right)$ ).

Definition 1. A proof of unsatisfiability $P$ for a set of clauses $C$ is a directed acyclic graph $\left(V_{P}, E_{P}\right)$, where $V_{P}$ is a set of clauses, such that

- for every vertex $c \in V_{P}$, either

- $c \in C$, and $c$ is a root, or

- $c$ has exactly two predecessors, $c_{1}$ and $c_{2}$, such that $c$ is the resolvent of $c_{1}$ and $c_{2}$, and

- the empty clause is the unique leaf.

Theorem 1. If there is a proof of unsatisfiability for clause set $C$, then $C$ is unsatisfiable.

Proof. By induction over the depth of the DAG, and transitivity of implication, every clause is implied by the conjunction of $C$, hence $C$ implies the empty clause (i.e., false), and is thus unsatisfiable.

Now we consider how a standard SAT solver might be modified to produce proofs of unsatisfiability. While searching for a satisfying assignment, a DPLL solver makes decisions, or arbitrary truth assignments to variables, and generates from these an implication graph. This is a directed acyclic graph whose vertices are truth assignments to variables, where each node is implied by its predecessors in the graph together with single clause.

As an example, suppose that our clause set is $\{(\neg a \vee b),(\neg b \vee c \vee d)\}$ and we have already decided the literals $\{a, \neg c\}$. A possible implication graph is shown below:

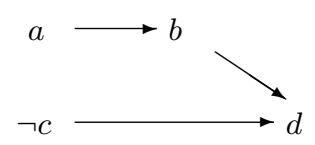

The literal $b$ is implied by node $a$ and the clause $(\neg a \vee b)$, while $d$ is implied by the nodes $b, \neg c$, and clause $(\neg b \vee c \vee d)$.

A clause is said to be in conflict when the negations of all its literals appear in the implication graph. When a conflict occurs, the SAT solver generates a conflict clause - a new clause that is implied by the existing clauses in the set. This is usually explained in terms of finding a cut in the implication graph, but from our point of view it is better understood as a process of resolving the "clause in conflict" with clauses in the implication graph to generate a new clause (that is also in conflict). We can also think of each resolution step as applying an implication from the implication graph in the contrapositive. 
As an example, suppose that we add the clause $(\neg b \vee \neg d)$ to the example above. This clause is in conflict, since the implication graph contains both $b$ and $d$. Note that $d$ was implied by the clause $(\neg b \vee c \vee d)$. Taking the resolvent of this clause with the conflicting clause $(\neg b \vee \neg d)$, we obtain a new implied clause $(\neg b \vee c)$, which is also in conflict. Now, the literal $b$ in the implication graph was implied by the clause $(\neg a \vee b)$. Resolving this with our new clause produces another implied clause $(\neg a \vee c)$, also in conflict. Either of these implied clauses might be taken as the conflict clause, and added to the clause set.

In order to generate a proof in the unsatisfiable case, we have only to record, for each generated conflict clause, the sequence of clauses that were resolved to produce that clause. The SAT solver produces an "unsatisfiable" answer when it generates the empty clause as a conflict clause (actually, most solvers do not explicitly produce this clause, but can be made to do so). At this point, we can easily produce a proof of unsatisfiability by, for example, a depth-first search starting from the empty clause, recursively deducing each clause in terms of the sequence of clauses that originally produced it. Note that, in general, not all conflict clauses generated during the SAT procedure will actually be needed to derive the empty clause.

\section{Proof-Based Abstraction}

Now we will show how such proofs of unsatisfiability can be used to generate abstractions for model checking. What follows does not rely on the fact that we are using a DPLL-style SAT solver. Any solver which can produce a proof of unsatisfiability will suffice, although the quality of the abstraction depends on the quality of the proof.

Bounded model checking [3] is a technique for proving that a transition system admits no counterexample to a given temporal formula of $k$ or fewer transitions, where $k$ is a fixed bound. This can be accomplished by posing the existence of a counterexample of $k$ steps or fewer as a SAT problem. Note that with a proof-generating SAT solver, in the unsatisfiable case we can in effect extract a proof of the non-existence of a counterexample of length $k$. This proof can in turn be used to generate an abstraction of the transition system in a very straightforward way.

We first observe that a bounded model checking problem consists of a set of constraints - initial constraints, transition constraints, final constraints (in the case of safety properties) and fairness constraints (conditions that must occur on a cycle, in the case of a liveness property). These constraints are translated into conjunctive normal form, and, if appropriate, instantiated for each time step $1 \ldots k$. If no clause derived from a given constraint is used in the proof of unsatisfiability, then we can remove that constraint from the problem, without invalidating the proof. Thus, the resulting abstract system (with unused constraints removed) is also guaranteed to admit no counterexample of $k$ steps or fewer. 
We can now apply ordinary (unbounded) model checking to the abstracted system. This process will have two possible outcomes. The first is that the property is true in the abstracted system. In this case, since removing constraints preserves all properties of our logic (linear temporal logic) we can conclude that the property is true in the original system and we are done. The second possibility is that the unbounded model checker will find a counterexample of greater than $k$ transitions (say, $k^{\prime}$ transitions). Note that a counterexample of fewer transitions is ruled out, since we have a proof that no such counterexample exists (in both the original or the abstracted system). In this case, we can simply return to bounded model checking using $k^{\prime}$ as the new length bound.

This procedure, which alternates bounded and unbounded model checking, is guaranteed to terminate for finite models, since $k$ is always increasing. At some point, $k$ must be greater than the depth of the abstract state space (i.e., the depth of a breadth-first search starting from the initial states). At this point, if there is no counterexample of length $k$, there can be no counterexample of length greater than $k$, thus the unbounded model checking step must yield "true". In practice, we usually find that when the procedure terminates, $k$ is roughly half the depth of the abstract state space.

In this procedure, "false" results (i.e., counterexamples) are only found by bounded model checking - counterexamples produced by the unbounded model checker are discarded, and only their length is taken into account in the next iteration. This is in contrast to counterexample-based methods such as [12 15, 92216] in which the counterexample produced by model checking the abstract system is used as a guide in refining the abstraction.

Also note that the set of constraints in the abstraction is not strictly growing with each iteration, as it is the above cited methods. That is, at each iteration, the old abstraction is discarded and a new one is generated based on the proof extracted from the SAT solver. This new abstraction may not contain all of the constraints present in the previous abstractions, and may even have fewer constraints.

In the remainder of this section, we will endeavor to make the above informal discussion more precise. Our goal is to determine whether a given LTL formula is true in a given finite model. However, this problem will be posed in terms of finding an accepting run of a finite automaton. The translation of LTL model checking into this framework has been extensively studied [15|21|10], and will not be described here. We will treat only safety properties here, due to space considerations. Liveness properties are covered in 13 .

\subsection{Safety Checking Algorithm}

For safety properties, we wish to determine the existence of a bad finite prefix - a finite sequence which cannot be extended to an infinite sequence satisfying the property. We assume that the problem is given in terms of an automaton on finite words, such that a bad prefix exists exactly when the automaton has an accepting run. Such a construction can be found, for example, in [11]. 
As in symbolic model checking, the automaton itself will be represented implicitly by Boolean formulas. The state space of the automaton is defined by an indexed set of Boolean variables $V=\left\{v_{1}, \ldots, v_{n}\right\}$. A state $S$ is a corresponding vector $\left(s_{1}, \ldots, s_{n}\right)$ of Boolean values. A state predicate $P$ is a Boolean formula over $V$. We will write $P(W)$ to denote $P\left\langle w_{i} / v_{i}\right\rangle$ (that is, $p$ with $w_{i}$ substituted for each $v_{i}$ ). We also assume an indexed set of "next state" variables $V^{\prime}=\left\{v_{1}^{\prime}, \ldots, v_{n}^{\prime}\right\}$, disjoint from $V$. A state relation $R$ is a Boolean formula over $V$ and $V^{\prime}$. We will write $R\left(W, W^{\prime}\right)$ to denote $R\left\langle w_{i} / v_{i}, w_{i}^{\prime} / v_{i}^{\prime}\right\rangle$.

The runs of the automaton are defined by a triple $M=(I, T, F)$, where the initial constraint $I$ and final constraint $F$ are state predicates, and the transition constraint $T$ is a state relation. A run of $M$, of length $k$, is a sequence of states $s_{0} \ldots s_{k}$ such that $I\left(s_{0}\right)$ is true, and for all $0 \leq i<k, T\left(s_{i}, s_{i+1}\right)$ is true, and $F\left(s_{k}\right)$ is true. We can translate the existence of a run into a Boolean satisfiability problem by introducing a new indexed set of variables $W_{i}=\left\{w_{i 1}, \ldots, w_{i n}\right\}$, for $0 \leq i \leq k$. A run of length up to $k$ exists exactly when the following formula is satisfiable 1

$$
I\left(W_{0}\right) \wedge\left(\bigwedge_{0 \leq i<k} T\left(W_{i}, W_{i+1}\right)\right) \wedge\left(\bigvee_{0 \leq i \leq k} F\left(W_{i}\right)\right)
$$

In order to use a standard SAT solver, we must translate this formula into conjunctive normal form. For this purpose, we will assume that $I$ and $T$ are each a conjunction of a collection of terms. That is, $I=\bigwedge_{j} I_{j}$ and $T=\bigwedge_{j} T_{j}$. This decomposition will allow us to abstract the problem by removing irrelevant terms. Further, to simplify matters, we can assume without loss of generality that the final condition $F$ consists of a single literal. To ensure this we can, for example, create a new state variable corresponding to the formula $F$ and fold the definition of this new variable into $T$.

We also assume the existence of some function $\Gamma$ that translates each Boolean formula into a logically equivalent set of clauses. Thus satisfiability of the above formula is equivalent to satisfiability of the following set of clauses:

$$
\operatorname{BMC}_{k}(M)=\left(\bigcup_{j} \Gamma\left(I_{j}\left(W_{0}\right)\right)\right) \cup\left(\bigcup_{0 \leq i<k, j} \Gamma\left(T_{j}\left(W_{i}, W_{i+1}\right)\right)\right) \cup\left\{\bigvee_{0 \leq i \leq k} F\left(W_{i}\right)\right\}
$$

Also note that in general the translation of an arbitrary Boolean formula $f$ into $\mathrm{CNF}$ is exponential. In practice, the problem can be solved by adding a fresh variable for the value of each subformula of $f$, as in [17. This construction does not affect the satisfiability of the result formula, and produces a CNF formula which is linear size in the size of $f$. The theory that follows, however, does not depend on the manner in which translation to CNF is performed.

At this point, if $\mathrm{BMC}_{k}(M)$ is found to be satisfiable, then we have a finite counterexample, and we are done. If, on the other hand, a proof of unsatisfiability

\footnotetext{
${ }^{1}$ Actually, this is correct only when the transition relation is total. The generalization to partial transition relations is straightforward.
} 
$P$ is found for $\operatorname{BMC}_{k}(M)$, then we know only that there is no counterexample of $k$ or fewer transitions. In this case, we build an abstraction $M^{\prime}$ of $M$, such that $P$ is also a proof of unsatisfiability of $\mathrm{BMC}_{k}\left(M^{\prime}\right)$. We want $\mathrm{BMC}_{k}\left(M^{\prime}\right)$ to retain all of the clauses used in $P$. Thus, we let $I^{\prime}$ be the conjunction of all the components $I_{j}$ such that some clause in $\Gamma\left(I_{j}\left(W_{0}\right)\right)$ occurs in $P$. Similarly, we let $T^{\prime}$ be the conjunction of all the components $T_{j}$ such that for some $0 \leq i<k$, some clause in $\Gamma\left(T_{j}\left(W_{i}\right)\right)$ occurs in $P$. We need not abstract $F$ itself, since we assume that $F$ is a single literal. This gives us the following result:

Lemma 1. Let $M=(I, T, F)$, let $P$ be a proof of unsatisfiability of $\operatorname{BMC}_{k}(M)$ and let $M^{\prime}=\left(I^{\prime}, T^{\prime}, F^{\prime}\right)$ where

$-I^{\prime}=\bigwedge\left\{I_{j} \mid \Gamma\left(I_{j}\left(W_{0}\right)\right) \cap V_{P} \neq \emptyset\right\}$,

$-T^{\prime}=\bigwedge\left\{T_{j} \mid\left(\bigcup_{0 \leq i<k} \Gamma\left(T_{j}\left(W_{i}\right)\right)\right) \cap V_{P} \neq \emptyset\right\}$, and

$-F^{\prime}=F$.

$M^{\prime}$ has no runs of length $k$ or less, and further, if $M^{\prime}$ has no runs, then $M$ has no runs.

Proof. By definition, every clause in $P$ that occurs in $\mathrm{BMC}_{k}(M)$ also occurs in $\mathrm{BMC}_{k}\left(M^{\prime}\right)$. Thus, since $P$ is a proof of unsatisfiability of $\mathrm{BMC}_{k}(M)$, it is also a proof of unsatisfiability of $\mathrm{BMC}_{k}\left(M^{\prime}\right)$, so the abstraction $M^{\prime}$ also has no run of length $k$ or less. Further, since $I^{\prime}, T^{\prime}$ and $F^{\prime}$ are weaker than $I, T$ and $F$, respectively, it follows that every run of $M$ is also a run of $M^{\prime}$.

We can now attempt to perform unbounded symbolic model checking on $M^{\prime}$, to determine whether it has a run of any length. This is preferable to applying model checking directly to $M$ in the case when the number of variables referenced in $M^{\prime}$ is significantly smaller than the number referenced in $M$, yielding a reduction in the effective size of the state space. If model checking of $M^{\prime}$ determines that $M^{\prime}$ has no runs, then $M$ has no runs, and we are done. On the other hand, if $M^{\prime}$ does have a run, we know that its length $k^{\prime}$ is greater than $k$. In this case, we restart the procedure with $k^{\prime}$ for $k$ (or in general, any value larger than $k^{\prime}$ for $k$ ). The overall procedure is shown in figure 1.

Theorem 2. If $M$ has a run, then FiniteRun(M) terminates and returns a run of $M$, else it terminates and returns "No Run".

Proof. Suppose, toward a contradiction, that the procedure does not terminate. Then, by lemma 1, $k$ increases without bound. Thus, if a run of $M$ does exist, eventually $B M C_{k}(M)$ will be satisfiable, and the procedure will terminate, returning a run. On the other hand, if $M$ has no run, then eventually $k$ will exceed $2^{n}$, where $n$ is the number of state variables. At this point, since $M^{\prime}$ has no runs of length up to $2^{n}$ (an upper bound on the depth of its state space) it has no runs. Hence the procedure terminates, returning "No Run".

A number of optimizations can be applied to this basic method in order to produce smaller abstract models. These include a "cone of influence" reduction on the abstract model, as well as methods to reduce the number of free combinational variables and to improve the proofs generated by the SAT solver. These are omitted here due to space considerations, but are described in [13]. 


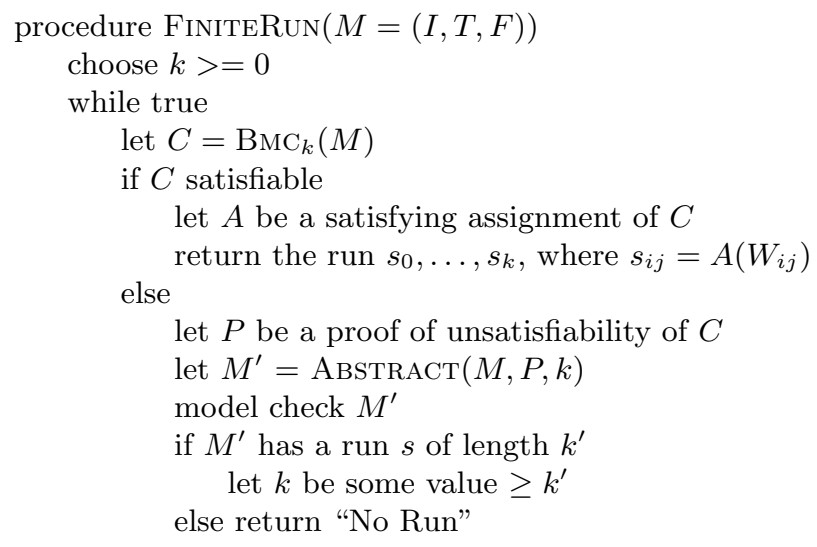

end

procedure $\operatorname{Abstract}\left(M=(I, T, F), P=\left(V_{P}, E_{P}\right), k\right)$

let $I^{\prime}=\bigwedge\left\{I_{j} \mid \Gamma\left(I_{j}\left(W_{0}\right)\right) \cap V_{P} \neq \emptyset\right\}$

let $T^{\prime}=\bigwedge\left\{T_{j} \mid\left(\bigcup_{0 \leq i<k} \Gamma\left(T_{j}\left(W_{i}\right)\right)\right) \cap V_{P} \neq \emptyset\right\}$

return $\left(I^{\prime}, T^{\prime}, F\right)$

end

Fig. 1. Procedure for existence of a finite run

\section{Practical Experience}

A direct comparison of the proof-based abstraction method against counterexample based methods such as [226 16] is unfortunately not possible, since the performance data presented in these works is based on proprietary benchmark problems (also, the most closely related work [16] appeared after this paper was submitted).

To guage the effectivenss of the proof-based abstraction procedure in generating abstractions, it was tested on a set of benchmark model checking problems derived from a sampling of properties used in the compositional verification of a unit of the PicoJava II microprocessor, available in open source from Sun Microsystems, Inc 2 The unit in question is the ICU, which manages the instruction cache, prefetches instructions, and does some preliminary instruction decoding. Originally, the properties were verified by standard symbolic model checking, using some manual directives to remove parts of the logic not relevant to each property. To make interesting benchmark examples for automatic abstraction, these directives were removed, and a neighboring unit, the instruction folding unit (IFU) was added. The intention of this is to simulate the actions of a naïve user who is unable to localize the verification problem manually (the ultimate

\footnotetext{
2 The tools needed to construct the benchmark examples from the Pico-Java II source code can be found at http://www-cad.eecs.berkeley.edu/ ${ }^{\sim}$ kenmcmil.
} 
naïve user being an automated tool). The function of the IFU is to read instruction bytes from the instruction queue, parse the byte stream into separate instructions and divide the instructions into groups that can be fed into the execution unit in a single cycle. Inclusion of the IFU increases the number of state variables in the "cone of influence" substantially, largely by introducing dependencies on registers within the ICU itself. It also introduces a large amount of irrelevant combinational logic.

Twenty representative properties were chosen as benchmarks. All of these properties are safety properties, of the form $G p$, where $p$ is a formula involving only the current time and the next time (usually only the current time). All the properties are true. Tests were performed on a Linux workstation with a $930 \mathrm{MHz}$ Pentium III processor and 512MB of available memory. Unbounded symbolic model checking was performed using the Cadence SMV system. SAT solving was performed using an implementation of the CHAFF algorithm [14, modified to produce proofs of unsatisfiability (verification using a modification of the actual Princeton zChaff implementation produced substantially similar results).

None of the benchmarks could be successfully verified by standard symbolic model checking methods, within a limit of 1800 seconds 3

On the other hand, of the 20 benchmarks, all but two were successfully verified by the proof-based abstraction technique. In the two failed cases, the failure was caused by memory exhaustion by the SAT solver during the bounded model checking phase (at $k$ values of 15 and 20 transitions, respectively). Notably, in all cases where the bounded model checking phase completed successfully, the unbounded symbolic model checker was able to successfully check the resulting abstraction $M^{\prime}$.

Figure 2 shows, for each benchmark, the original number of state holding variables (solid bars), the number obtained by manual abstraction (gray bars) and the number of state variables remaining in the abstraction at the final iteration of the proof-based abstraction algorithm, without manual abstraction (open bars). Here, by state variables, we mean any variable $v$ such that $v^{\prime}$ occurs in $T$. We will refer to other variables, including inputs and intermediate variables as "combinational variables". A $\otimes$ below the bars indicates that the algorithm did not complete. The number of variables obtained by manual abstraction does not necessarily reflect what could be obtained by concerted effort, but rather reflects only a sufficient effort to make the properties checkable by standard methods. Nonetheless, it is interesting to note that in 11 out of 20 cases a better result is obtained by automatic abstraction.

Figure 3 shows total run time of the proof-based abstraction procedure for each of the benchmarks, on a log scale. Comparison data are not available for standard symbolic model checking, since no problem could be completed within

\footnotetext{
${ }^{3}$ The primary cause of this failure appears to be inability to construct BDD's for parts of the combinational logic in the IFU. It is possible that some of the benchmarks could be completed by using more advanced transition relation decomposition techniques than are implemented in Cadence SMV.
} 


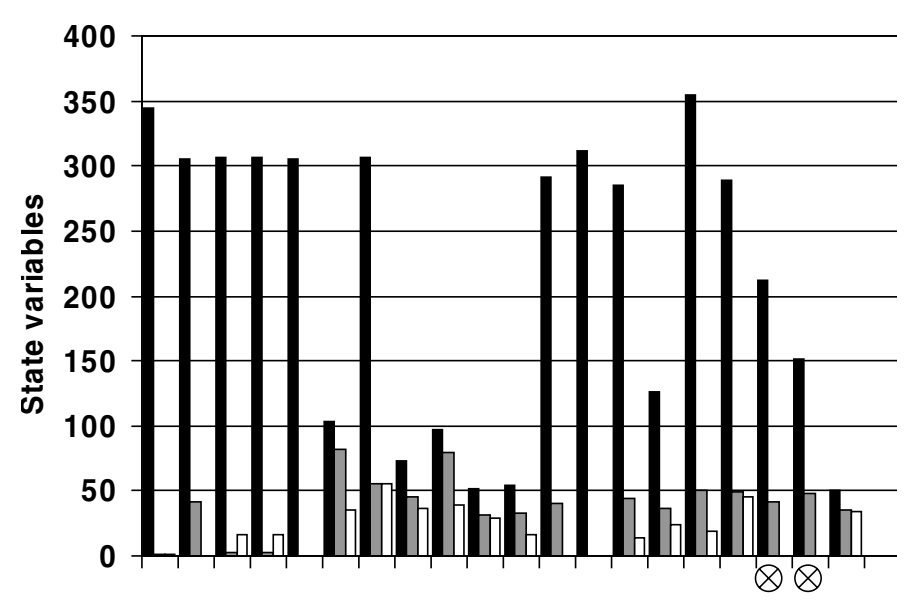

Fig. 2. State variables: (solid) original, (gray) after manual abstraction, (open) after automatic abstraction.

the allotted time. Figure 4 shows the fraction of total run time spent in the two phases of the algorithm. The solid part of the bars represent the total time spent in the bounded model checking phase, while the open part represents the total time spent in the unbounded model checking phase. Note that in most cases, the bottleneck is bounded model checking.

What these data clearly show is that the SAT solver is effective at isolating the part of the logic that is relevant to the given property, at least in the case when this part of the logic is relatively small. We have also found the technique to be very effective at falsification, since the unbounded model checking phase quickly guides the bounded model checker to the appropriate depth.

As an additional point of comparison, figure 5 compares the performance of the proof-based abstraction approach with results previously obtained by Baumgartner et al. 2] on a set of benchmark model checking problems derived from the IBM Gigahertz Processor. Their method involved a combination of SAT-based bounded model checking, structural methods for bounding the depth of the state space, and target enlargement using BDD's. Each point on the graph represents the average verification or falsification time for a collection of properties of the same circuit model. The average time in seconds for proofbased abstraction is represented on the $\mathrm{X}$ axis, while the average time in seconds obtained by Baumgartner et al. is represented on the $\mathrm{Y}$ axis 4 Thus, a point above the diagonal line represents a lower average time for proof-based abstraction for one benchmark. Note that in several cases proof-based abstraction has an advantage of two orders of magnitude. A time of 1000 seconds indicates that

\footnotetext{
${ }_{4}^{4}$ The processor speeds for the two sets of experiments are slightly different. Baumgartner et al. used an $800 \mathrm{MHz}$ Pentium III, as compared to a $930 \mathrm{MHz}$ Pentium III used here. The results presented here have not been adjusted to reflect CPU speed.
} 


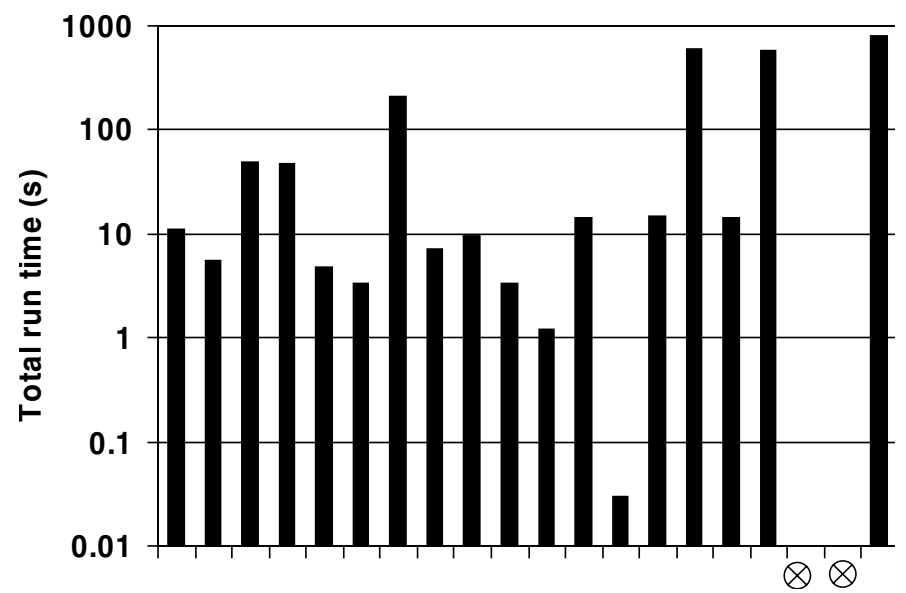

Fig. 3. Total verification time for proof-based abstraction algorithm.

the truth of one or more properties in the benchmark could not be determined. Of the 28 individual properties that could not be resolved by Baumgartner et al., all but one are successfully resolved by the proof-based abstraction method. Excluding this failed benchmark, the largest average time for the proof-based method is 2.89 seconds. The clear conclusion is that proof-based abstraction is a more effective method of exploiting a SAT solver for model checking.

\subsection{A Hypothesis}

The fact that the unbounded model checker is able to check the abstraction in most cases when the bounded model checking succeeds suggests an interesting (if somewhat informal) hypothesis: that is, that bounded model checking using SAT solvers tends to succeed when the number of relevant variables is small, and to fail when the number of relevant variables is large. Thus far we have tested only the case when the number of relevant variables is small. To test the other end of the spectrum, one possible approach is to use a set of scalable benchmarks, in which all or most of the state variables are known to be relevant. Such examples tend to occur, for example, in protocol verification. Here, absent any fault tolerance mechanism, a dropped bit anywhere in the system tends to cause the protocol to fail.

We will consider first a simple model of a cache coherence protocol due to Steven German [8]. This model is parameterized by $N$, number of processors. The property to be proved is that, if there is an "exclusive" copy of a cache line in the system, then there is no other copy. Empirically, the the depth of the state space of this model is found to be $8 N+2$ transitions. Applying bounded model checking to the model at this depth, we find that the largest instance of this problem we can solve within 1800 seconds is $N=4$, which has only 42 state variables (of which 37 are found to be relevant). This is quite surprising considering the 


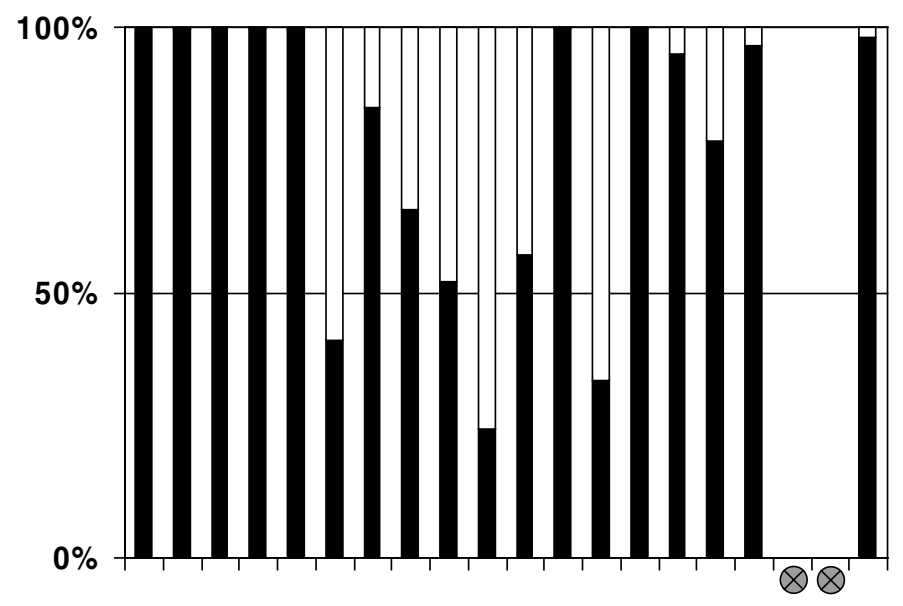

Fig. 4. Fraction of total run time: (solid) bounded model checking (open) unbounded model checking

extreme simplicity of this model relative to the PicoJava II benchmarks. In that case, the SAT solver managed to solve CNF SAT problems with on the order of 1 million variables, while in this case it fails with only about 40,000 variables. On the other hand, the number of relevant state holding variables is roughly similar to what was handled in the PicoJava II benchmarks.

As another test case, let us consider a simple circuit we will call swap. This circuit has $n j$-bit registers. At each clock cycle, it inputs a number $i$, and swaps the values of register $i$ and its neighbor $i+1 \bmod j$. We set the number of bits $j$ to $\left\lceil\log _{2} n\right\rceil$ so that we can initialize all of the registers to different values. The property to prove is that registers 0 and 1 always differ. Clearly, if we unconstrain the value of any one register, the property will be false, since by a series of swaps, we can transfer the value of any register to register 0 . Interestingly, we find that the largest instance of swap that we can successfully apply bounded model checking to is $n=7$, corresponding to 21 state bits. At $n=8$, and $k=8$, the zChaff solver failed to solve a SAT problem with only 1396 variables in over 40 hours!

Testing SAT solvers on other hardware designs tends to confirm the following trend: when proofs are successfully produced by the SAT solver, they tend to involve only a small number of variables in an absolute sense. Figure 6] shows results on the set of problems in a collection of hardware verification benchmarks used at Cadence Design Systems. Each point represents a single benchmark problem, with the $\mathrm{X}$ axis giving the original number of state variables, and the $\mathrm{Y}$ axis the number of state values in the abstraction resulting from the longest successful bounded model checking run in the proof-based abstraction procedure. The trend is clear: successful bounded model checking runs tend to produce proofs of unsatisfiability using a small number of state variables, 


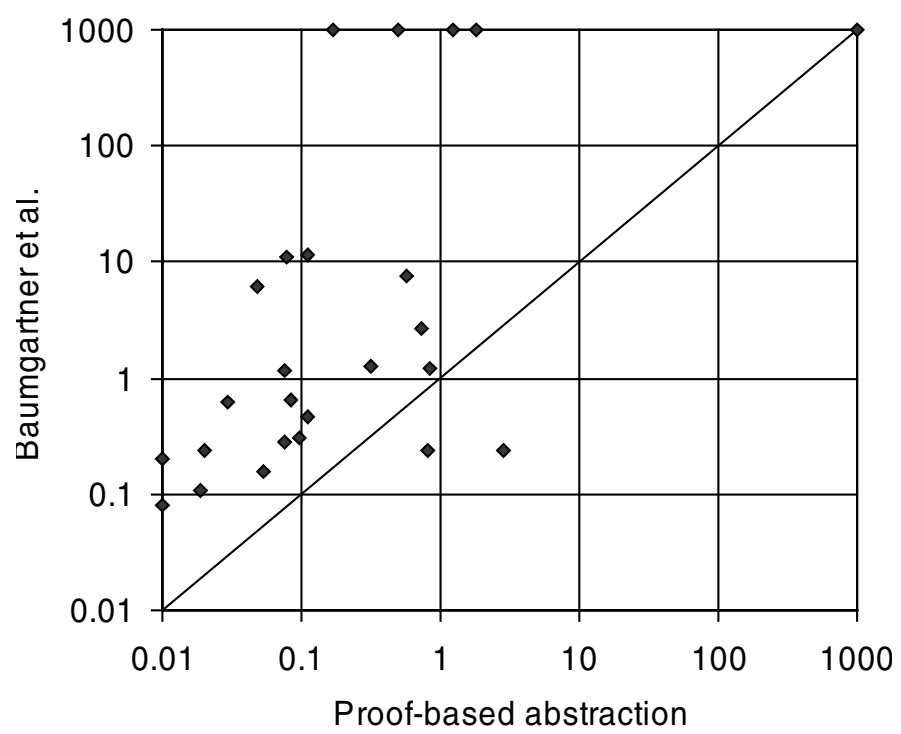

Fig. 5. Fraction of total run time: (solid) bounded model checking (open) unbounded model checking

independent of the number of original state variables. In 18 out of 20 cases, the BDD-based model checker is able to check the abstraction. On the other hand, a very large example provided by IBM produced a proof-based abstraction with over 1000 state bits. We conjecture that this was possible because a large number of these registers do not leave their initial states within the first $k$ steps, and that the SAT solver did not in fact reach the state space depth. This has not been confirmed, however.

On the whole, while the case studies presented here are certainly too small to draw general conclusions about the performance of bounded model checking, they are consistent with the hypothesis that successful bounded model checking (defined as checking up to the state space depth) depends on having small number of relevant state variables. This suggests that a larger scale study of the question might be in order.

\section{Conclusion}

We have observed that information generated by bounded model checking can be used to improve the efficiency of unbounded model checking, by suggesting abstractions. Perhaps more interestingly, we have seen some empirical evidence for the hypothesis that bounded model checking succeeds when the number of relevant state variables is small, which implies that unbounded model checking is also likely to succeed when applied to the relevant parts of the system. If this hypothesis holds true generally, then it may prove unnecessary in practice to 


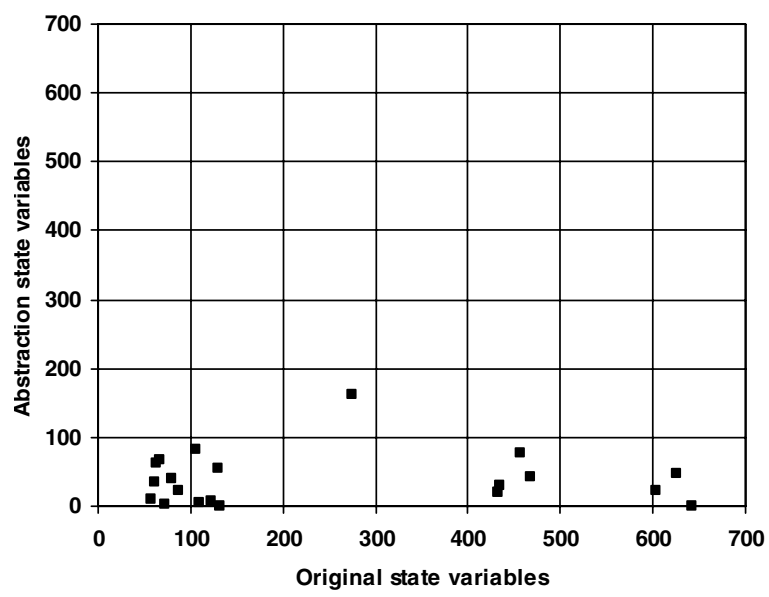

Fig. 6. Results of proof-based abstraction on hardware benchmarks.

find techniques for bounding the search depth in bounded model checking - if bounded model checking succeeds at roughly the state space depth, then the result can likely be confirmed by standard model checking.

Of course, the negative side of this observation is that it seems unlikely that bounded model checking (and hence proof-based abstraction) can be applied to global properties of systems (those that depend on most or all of the state variables). Techniques are still required to reduce the verification problem to "local" properties that can be proved using only a small set of state variables. However, SAT solvers appear to have significant potential for identifying that set of variables once a suitable property is given.

For future work, it is interesting to consider what other information can be extracted from proofs of unsatisfiability that might be useful in model checking. In addition, since SAT solvers seem to be so effective at isolating relevant facts, it might be that a similar technique could also be applied to infinite state methods such as predicate abstraction, by means of various translations from first order to Boolean satisfiability problems. A final interesting avenue of research might be to consider how the basic SAT algorithms might be modified to improve their performance in terms of producing compact proofs, which would lead in turn to better abstractions.

\section{References}

1. F. Balarin and A. Sangiovanni-Vincentelli. An iterative approach to language containment. In Computer Aided Verification (CAV'93), pages 29-40, 1993.

2. J. Baumgratner, A. Kuehlmann, and J. Abraham. Property checking via structural analysis. In Computer-Aided Verification (CAV 2002), pages 151-165, 2002.

3. A. Biere, A. Cimatti, E. Clarke, and Y. Zhu. Symbolic model checking without BDDs. In TACAS'99, volume 1579 of $L N C S$, pages 193-207, 1999. 
4. P. Bjesse, T. Leonard, and A. Mokkedem. Finding bugs in an alpha microprocessor using satisfiability solvers. In Computer Aided Verification (CAV 2001), 2001.

5. E. M. Clarke, O. Grumberg, S. Jha, Y. Lu, and H. Veith. Counterexample-guided abstraction refinement. In Computer Aided Verification, pages 154-169, 2000.

6. E. M. Clarke, A. Gupta, J. Kukula, and O. Strichman. SAT based abstractionrefinement using ILP and machine learning techniques. In Computer-Aided Verification (CAV 2002), pages 265-279, 2002.

7. F. Copty, L. Fix, F. R, E. Giunchiglia, G. Kamhi, A. Tacchella, and M. Y. Vardi. Benefits of bounded model checking in an industrial setting. In Computer Aided Verification (CAV 2001), pages 436-453, 2001.

8. S. German. Personal communication.

9. S. G. Govindaraju and D. L. Dill. Counterexample-Guided choice of projections in approximate symbolic model checking. In IEEE International Conference on Computer Aided Design (ICCAD 2000), pages 115-119, 2000.

10. J.R. Burch, E.M. Clarke, K.L. McMillan, D.L. Dill, and L.J. Hwang. Symbolic Model Checking: $10^{20}$ States and Beyond. In Proceedings of the Fifth Annual IEEE Symposium on Logic in Computer Science, pages 1-33, Washington, D.C., 1990. IEEE Computer Society Press.

11. O. Kupferman and M. Y. Vardi. Model checking of safety properties. Formal Methods in System Design, 19(3):291-314, 2001.

12. R. P. Kurshan. Computer-Aided-Verification of Coordinating Processes. Princeton University Press, 1994.

13. K. L. McMillan and N. Amla. Automatic abstraction without counterexamples. http://www-cad.eecs.berkeley.edu/ ${ }^{\sim}$ kenmcmil/papers, 2002.

14. M. W. Moskewicz, C. F. Madigan, Y. Z., L. Z., and S. Malik. Chaff: Engineering an efficient SAT solver. In Design Automation Conference, pages 530-535, 2001.

15. A. P. O. Lichtenstein. Checking that finite state concurrent programs satisfy their linear specification. In POPL '85, pages 97-107, 1985.

16. J. K. S. S. H. V. Pankaj Chauhan, Ed Clarke and D. Wang. Automated abstraction refinement for model checking large state spaces using sat based conflict analysis. In Formal Methods in Computer Aided Design (FMCAD'02), November 2002.

17. D. Plaisted and S. Greenbaum. A structure preserving clause form translation. Journal of Symbolic Computation, 2:293-304, 1986.

18. H. Saïdi and S. Graf. Construction of abstract state graphs with PVS. In O. Grumberg, editor, Computer-Aided Verification, CAV '97, volume 1254, pages 72-83, Haifa, Israel, 1997. Springer-Verlag.

19. J. P. M. Silva and K. A. Sakallah. GRASP-a new search algorithm for satisfiability. In Proceedings of the International Conference on Computer-Aided Design, November 1996, 1996.

20. R. M. T. A. Henzinger, R. Jhala and G. Sutre. Lazy abstraction. In Principles of Programming Languages (POPL 2002), 2002.

21. M. Vardi and P. Wolper. An automata-theoretic approach to automatic program verification. In Logic in Computer Science (LICS '86), pages 322-331, 1986.

22. D. Wang, P.-H. Ho, J. Long, J. H. Kukula, Y. Zhu, H.-K. T. Ma, and R. Damiano. Formal property verification by abstraction refinement with formal, simulation and hybrid engines. In Design Automation Conference, pages 35-40, 2001. 